\section{Presença e extensão dos atributos de atenção primária à saúde da criança em distintos modelos de cuidado}

\section{Presence and extent of primary care characteristics under different models for children's healthcare}

\author{
Presencia y extensión de las particularidades de \\ la atención primaria, relacionadas con la salud \\ del niño, en distintos modelos de cuidado
}

\begin{abstract}
Resumo
O objetivo deste estudo é avaliar qual modelo de atenção, entre unidade básica de saúde (UBS) tradicional e aquelas com Estratégia Saúde da Família (ESF), é mais orientado à atenção primária à saúde (APS) da criança, considerando a presença e a extensão dos atributos essenciais e derivados da APS. Foram entrevistados 1.484 familiares e ou cuidadores de crianças menores de 10 anos de idade cadastradas em unidades de saúde da família de João Pessoa na Paraíba, Brasil, e UBS tradicionais de Cascavel, e UBS mistas de Londrina, ambas no Paraná, Brasil. Para tanto, utilizou-se o Primary Care Assessment Tool Brasil, versão criança. Para análise utilizou-se o teste ANOVA one way paramétrica, para identificação de diferença estatisticamente significante entre os modelos de atenção, seguida do teste de comparação múltipla (post hoc) de Tukey, para apontar qual modelo apresentava diferença. Apesar de limitações no desenho do estudo, os achados levantam a hipótese de que a UBS mista é o modelo mais orientado à APS no cuidado à criança. Isso pode estar relacionado ao fato de que os diferentes modelos avaliados pertenciam a municípios diferentes, sem possibilidade de ajustar, na análise, as variáveis ligadas aos diferentes contextos. Assim, estudos futuros, com desenhos mais robustos, e ampliando para outros usuários e profissionais de saúde, poderão comprovar esta hipótese.
\end{abstract}

Atenção Primária à Saúde; Saúde da Criança; Pesquisa sobre Serviços de Saúde
Nathanielly Cristina Carvalho de Brito Santos 1,2 Elenice Maria Cecchetti $\mathrm{Vaz}^{3}$

Jordana Almeida Nogueira 2

Beatriz Rosana Gonçalves de Oliveira Toso 4

Neusa Collet 2

Altamira Pereira da Silva Reichert 2

doi: 10.1590/0102-311X00014216

\author{
Correspondência \\ N. C. C. B. Santos \\ Centro de Educação e Saúde, Universidade Federal de Campina \\ Grande. \\ Rua Antonio Joaquim Pequeno 233, Campina Grande, PB \\ 58429-010, Brasil. \\ nathaniellycristina@gmail.com \\ 1 Centro de Educação e Saúde, Universidade Federal de \\ Campina Grande, Campina Grande, Brasil. \\ 2 Programa de Pós-graduação em Enfermagem, Universidade \\ Federal da Paraíba, João Pessoa, Brasil. \\ 3 Escola de Enfermagem Aurora de Afonso Costa, Universidade \\ Federal Fluminense, Niterói, Brasil. \\ 4 Programa de Pós-graduação em Biociências e Saúde, \\ Universidade Estadual do Oeste do Paraná, Cascavel, Brasil.
}




\section{Introdução}

A atenção à saúde da criança vem passando por transformações em função da mudança de perfil epidemiológico dessa população, dos avanços científicos, da incorporação de tecnologias, e da constante mudança nos modelos assistenciais, com apelo para a preocupação com a qualidade de vida e a defesa dos direitos humanos 1 .

Por isso, e também diante da maior vulnerabilidade em seu processo de crescimento e desenvolvimento, a criança é considerada uma prioridade nas políticas públicas de saúde. Tais políticas vêm evoluindo com foco na consolidação de uma assistência integral, por meio da oferta de ações e serviços de prevenção e promoção à saúde em consonância com a atenção primária à saúde (APS), buscando garantir a redução da morbimortalidade infantil por causas evitáveis e sua sobrevivência com qualidade de vida 2 .

Todavia, apesar do cuidado à criança buscar promover o acompanhamento sistemático do crescimento e desenvolvimento pela interação entre serviços-profissionais-família-comunidade, e um agir compartilhado frente à implementação das ações de prevenção, cura, reabilitação e promoção da saúde infantil 3, ainda é possível se deparar com um cuidado descoordenado, com ações fragmentadas e pouco resolutivas frente às demandas de saúde da criança e sua família, o que dificulta a integralidade do cuidado na APS 4 .

Por conseguinte, torna-se necessário refletir acerca do acesso e da oferta de serviços na rede de atenção à saúde, a valorização de tecnologias leves de cuidado, representadas pelo vínculo, acolhimento e humanização como elementos essenciais para a reformulação do espaço micropolítico do processo de trabalho e das práticas de saúde 2 em prol de um cuidado integral, efetivo e de qualidade à criança na atenção primária. Para tanto, o modelo de atenção à saúde precisa estar orientado por eixos estruturantes que, na literatura internacional, recebem o nome de atributos essenciais e derivados da APS 5,6.

Constituem atributos essenciais o acesso de primeiro contato (acessibilidade e uso do serviço de saúde para acompanhamento rotineiro ou a cada novo problema), longitudinalidade (existência de um aporte regular de atenção de modo contínuo, incluindo uma relação interpessoal intensa que expresse a confiança mútua entre usuários e profissionais), integralidade (prestação de um conjunto de ações e serviços de promoção, prevenção e recuperação pelas unidades de saúde ou encaminhados por elas, de modo a atender às necessidades de saúde da população adscrita) e coordenação (garantia da continuidade da atenção e reconhecimento dos problemas que necessitam de seguimento constante, bem como das informações a respeito de problemas e serviços anteriores que estão relacionadas às necessidades para o presente atendimento) 7 .

Como atributos derivados, e não menos importantes, pois qualificam as ações implementadas na APS, estão a orientação familiar (considera a família como sujeito da atenção com potencialidades para o cuidado) e orientação comunitária (reconhecimento das necessidades apresentadas pelas famílias em função do contexto geoeconômico e sociocultural em que vivem) 7 .

Esses atributos representam importantes indicadores de qualidade da atenção básica, servindo para analisar as potencialidades de diferentes modalidades presentes nos serviços de saúde. Permitem identificar o grau de orientação dos modelos de atenção à APS, possibilitando o cotejamento dos mesmos acerca da qualidade da atenção ofertada e sua efetividade diante das necessidades de saúde do indivíduo e da população ${ }^{8}$. Destaca-se aqui a população infantil pelas singularidades a serem consideradas pelos profissionais no cuidado ofertado à criança na APS.

No cenário brasileiro, além das unidades de saúde da família (USF) que direcionam ações e práticas para o cuidado à criança em busca da atenção integral, coexistem outros modelos de APS: as unidades básicas de saúde (UBS) tradicionais e as consideradas aqui como UBS mistas. As UBS tradicionais concentram a assistência à saúde na figura do médico com atendimento pela tríade de especialistas - clínico, ginecologista e pediatra -, além da equipe de enfermagem e odontologia; e as unidades mistas que articulam diferentes modelos na USF 9, integram os processos de trabalho da Estratégia Saúde da Família (ESF) com o modelo tradicional para a mesma população.

Estudo realizado no Paraná, Brasil 10, que comparou a presença e extensão dos atributos da APS no atendimento de crianças entre o modelo assistencial de UBS tradicional e com ESF evidenciou que o modelo com ESF se aproxima mais dos princípios da APS, embora tenha constatado limitações 
em ambos os modelos. Isso abre espaço para outras produções científicas que venham propiciar avaliações mais amplas, com cotejos regionais acerca do desempenho da APS como propósito para aperfeiçoar a assistência em todo o território brasileiro.

Ante o exposto, o objetivo deste artigo é avaliar qual modelo de atenção, entre UBS tradicional e aquelas com ESF, é mais orientado à APS da criança, tendo em conta a presença e a extensão dos atributos essenciais e derivados da atenção primária à saúde.

\section{Métodos}

Estudo transversal e avaliativo, de abordagem quantitativa, realizado no período de outubro de 2012 a fevereiro de 2013, em unidades de APS, em três municípios do Brasil, sendo dois na Região Sul (Cascavel e Londrina, Paraná), e um na Região Nordeste (João Pessoa, capital do Estado da Paraíba). Quanto aos serviços de atenção primária, na época da coleta de dados, em Cascavel, predominava a atenção no modelo tradicional, com 22 UBS; em Londrina, havia 40 UBS tradicionais junto com USF 11, aqui denominadas UBS mistas; e em João Pessoa, cuja APS é ofertada na totalidade por ESF. Integraram o estudo 53 USF no Distrito Sanitário III (DS III). O DS III foi escolhido por ser o maior dos distritos da rede de atenção básica de João Pessoa, com cobertura de 90,5\% das famílias adscritas naquela área, com equivalência de equipes quando comparado aos demais municípios.

Destaca-se que a infraestrutura das referidas unidades de saúde nos três municípios seguiu o modelo padrão para serviços assistenciais de saúde de acordo com a $R D C$ no 50 12. Ainda que haja diferenças culturais, sociais, demográficas e de políticas públicas que podem influenciar nos resultados, assumiu-se o risco na escolha pelos cenários, tendo em vista que, nestes municípios escolhidos, havia os modelos que o estudo optou por avaliar. Além disso, eles possuem similaridade nos Índices de Desenvolvimento Humano (IDH) (Cascavel: 0,782, e Londrina: 0,778, no Paraná, e João Pessoa: 0,763, na Paraíba) e de Gini (Cascavel: 0,41, e Londrina: 0,42, no Paraná, e João Pessoa: 0,50, na Paraíba) (Instituto Brasileiro de Geografia e Estatística. http://www.ibge.gov.br/estadosat, acessado em 30/ Jul/2016). Ademais, a implantação dos modelos de atenção à saúde está direcionada à população de baixa renda e com maior vulnerabilidade social, o que sugere semelhanças nas condições que refletem saúde, educação e renda, mesmo que sejam de regiões distintas. Assim sendo, sugere-se que tais aspectos podem ser considerados como um indicador de homogeneização da amostra e controle de vieses para escolha dos cenários da pesquisa.

A população estudada foi constituída por familiares (pai, mãe) e/ou cuidadores (avós, tios, cuidadores legais) de crianças menores de 10 anos de idade, atendidas nas referidas unidades, num período de seis meses anteriores à coleta de dados. Com base no número de atendimentos registrados às crianças, distribuídos entre os três municípios, foi calculada uma amostra de 1.484 participantes, por amostragem probabilística estratificada proporcional, adotando-se uma margem de erro de 2,51\% e nível de 95\% de confiança, sendo 531 participantes em Cascavel, 609 em Londrina e 344 em João Pessoa. É necessário ressaltar que, diante da ausência de resposta para determinados componentes do formulário de coleta de dados, houve variação no total da amostra analisada.

Para seleção dos participantes, utilizou-se a amostragem por conveniência na fila de espera para o atendimento, dessa forma, era convidado o último familiar e/ou cuidador da criança na fila de espera para atendimento, e, caso ele não aceitasse, era convidado o imediatamente anterior, até completar a cota amostral estabelecida para cada unidade. Foram incluídos familiares e/ou cuidadores residentes na área urbana dos municípios que apresentaram capacidade de entendimento, expressão e compreensão dos documentos apresentados. Logo, o participante deveria conhecer a unidade que iria avaliar, sendo incluído aquele que levou a criança para atendimento por, pelo menos, duas vezes anteriores àquela na qual aguardava atendimento.

Para coleta de dados, utilizou-se o instrumento Primary Care Assessment Tool (PCATool) versão criança 13, já validado no Brasil 14. Esse instrumento contém questões organizadas em componentes que avaliam a presença e a extensão dos atributos essenciais e derivados da APS identificados pelas letras de A a J, que mensuram o grau de afiliação do usuário com o serviço (A), os atributos essenciais (B-H) e os atributos derivados (I-J). Cabe destacar que nesta versão criança, a avaliação dos serviços ocorre pela percepção de familiares e/ou cuidadores das crianças, no que difere da versão adulto. 
No instrumento, as respostas são enunciadas por meio da escala tipo Likert, cujos valores variam de um a quatro para cada item que compõe o atributo, correspondendo a 1 "com certeza não"; 2 "provavelmente não"; 3 "provavelmente sim"; 4 "com certeza sim" e, além desses, o valor 9, para as respostas "não sei/não lembro" 15 . A aplicação do instrumento foi realizada nas salas de espera das unidades de saúde referentes a cada município.

Para avaliação do grau de orientação dos diferentes modelos de APS da criança, utilizou-se o referencial de Starfield 6, baseado nos atributos essenciais e derivados da APS, calculados segundo as orientações do Manual do instrumento PCATool-Brasil, versão criança 13. Pelo cálculo dos escores de cada atributo, chegou-se ao escore essencial da APS obtido pela soma do escore médio dos atributos essenciais mais o grau de afiliação: Escore Essencial $=(A+B+C+D+E+F+G+H) / 8$, e o escore geral da APS, pela soma do escore médio dos atributos essenciais e derivados mais o grau de afiliação: Escore $\mathrm{Geral}=(\mathrm{A}+\mathrm{B}+\mathrm{C}+\mathrm{D}+\mathrm{E}+\mathrm{F}+\mathrm{G}+\mathrm{H}+\mathrm{I}+\mathrm{J}) / 10$. Os escores alcançados para cada componente foram transformados em escore ajustado numa escala de 0-10, seguindo a fórmula: Escore Ajustado = (Escore obtido - 1) x 10/3. Nessa transformação, foram definidos como valores elevados ou satisfatórios os escores $\geq 6,6$ e baixo ou insatisfatório os escores $<6,6$, indicando que a presença e extensão dos atributos ou oferta deles pelos diferentes modelos de APS pesquisados refletem a qualidade da atenção ofertada.

Para armazenamento, processamento e análise, os dados foram digitados em dupla entrada com validação do processo, utilizando-se o programa Excel versão 7.0 (Microsoft Corp., Estados Unidos) e o pacote estatístico Statistical Package for Social Sciences (SPSS Inc., Chicago, Estados Unidos), versão 13.0. Para comparação dos escores dos atributos entre os modelos de APS e avaliação da existência de diferenças estatisticamente significantes, foi utilizado o teste de Kruskal-Wallis (ANOVA one way não paramétrica). Associado a ele, foi aplicado o teste de comparação múltipla (post hoc) de Dunnett para identificação do modelo que apresentava a diferença.

Para avaliação do modelo mais orientado à APS da criança, utilizou-se o teste de análise de covariância multivariada, seguido do teste de comparação múltipla (post hoc) de Tukey para apontar qual modelo apresentava diferença.

A pesquisa foi desenvolvida respeitando os preceitos éticos da Resolução no 466/2012, sob a aprovação do Comitê de Ética em Pesquisa da Universidade Oeste do Paraná (UNIOESTE), com anuência das Secretarias Municipais de Saúde de João Pessoa, na Paraíba e Cascavel e Londrina, no Paraná. O Termo de Consentimento Livre e Esclarecido foi assinado por todos os familiares e/ou cuidadores das crianças atendidas nos referidos modelos de APS.

\section{Resultados}

Os resultados permitem verificar a percepção de 1.484 familiares e/ou cuidadores de crianças menores de 10 anos sobre a qualidade da atenção ofertada pelos distintos modelos de APS. A Tabela 1 apresenta as medidas descritivas do Escore Essencial e do Escore Geral, por modelos de APS. Quanto ao Escore Essencial, nos três modelos (USF, UBS e UBS mista) o valor foi satisfatório à APS (escore $\geq$ 6,6), enquanto no Escore Geral somente a UBS mista alcançou o ponto de corte com escore 6,7.

Ademais, pode-se observar na Tabela 1 que em todos os modelos a amostra é considerada de tamanho adequado, o que permite comparar médias por meio de testes paramétricos mesmo com ausência de normalidade, evocando o teorema central do limite ou técnicas comparativas robustas, como o teste de Welch (Tabela 2), no lugar do tradicional teste F para ANOVA.

Na Tabela 2, constata-se que há influência do Escore Essencial e do escore geral sobre os modelos de APS $(\mathrm{p}<0,001)$. Todavia, após comparação por meio do teste de Tukey, existe diferença estatisticamente significativa entre os três modelos para o Escore Essencial e entre UBS mista e os outros dois modelos (USF e UBS tradicionais) quanto ao Escore Geral. Em ambos os escores o modelo de UBS mista apresentou maior valor de Escore Médio em relação aos demais modelos USF e UBS tradicionais (7,1 e 6,7, respectivamente).

Quanto à análise de covariância multivariada, aplicando-se o teste Lambda de Wilks (Tabela 3), verifica-se que há diferença significativa no vetor de médias do Escore Essencial e do Escore Geral por modelo de APS ( $\mathrm{p}<0,001$ ), mas que as covariáveis “idade, anos de estudo, renda familiar”, como impor- 


\section{Tabela 1}

Medidas descritivas, média e erro padrão, dos escores essencial e geral por modelo de atenção primária à saúde (APS).

\begin{tabular}{lccc}
\hline Escore/Modelo (APS) & $\mathbf{n}$ & Média & Erro padrão \\
\hline Essencial & & & \\
USF & 344 & 6,6 & 0,07 \\
UBS tradicional & 531 & 6,8 & 0,05 \\
UBS mista & 609 & 7,1 & 0,05 \\
Geral & & & \\
USF & 344 & 6,4 & 0,08 \\
UBS tradicional & 531 & 6,4 & 0,05 \\
UBS mista & 609 & 6,7 & 0,05 \\
\hline
\end{tabular}

Escore Essencial: soma dos atributos essenciais; Escore Geral: soma dos atributos essências mais os atributos derivados da APS; UBS: unidade básica de saúde; USF: unidade de saúde da família.

Fonte: banco de dados da pesquisa, 2016.

\section{Tabela 2}

Avaliar a relação de influência de cada escore sobre os modelos de atenção primária à saúde (USF, UBS tradicional e UBS mista).

\begin{tabular}{lcc}
\hline Escore & Teste Welch & Teste de Tukey \\
\hline Essencial & $<0,001$ & USF, UBS tradicional, UBS mista \\
Geral & $<0,001$ & USF, UBS tradicional, UBS mista \\
\hline
\end{tabular}

Escore Essencial: soma dos atributos essenciais; Escore Geral: soma dos atributos essenciais mais os atributos derivados; UBS: unidade básica de saúde; USF: unidade de saúde da família.

Fonte: banco de dados da pesquisa, 2016.

\section{Tabela 3}

Análise de covariância multivariada para a comparação dos modelos de atenção primária à saúde e covariáveis.

\begin{tabular}{lc}
\hline Variáveis & Lambda de Wilks (valor de $\mathbf{p}$ ) \\
\hline Escore Essencial & $<0,001$ \\
Escore Geral & \\
Covariáveis & \\
Idade do principal cuidador & 0,288 \\
Anos de estudo do principal cuidador & 0,045 \\
Renda familiar do cuidador & 0,861 \\
\hline
\end{tabular}

Fonte: banco de dados da pesquisa, 2016.

tantes fatores de confundimento entre os modelos de APS comparados, não influenciam variações significantes nos referidos escores ( $p>0,05)$, exceto os anos de estudo do principal cuidador $(\mathrm{p}<0,05)$.

Em relação aos atributos da APS, a Tabela 4 mostra a comparação entre os escores dos atributos essenciais e derivados, apresentados pelos modelos USF, UBS e UBS mista. Inicialmente, observouse que apenas os atributos grau de afiliação, acesso de primeiro contato - utilização, coordenação e 


\section{Tabela 4}

Avaliação dos atributos essenciais e derivados da atenção primária à saúde (APS) da criança em diferentes modelos de atenção.

\begin{tabular}{|c|c|c|c|c|c|}
\hline Atributos da APS/Modelos de APS & n válido & $\begin{array}{l}\text { Escore } \\
\text { médio }\end{array}$ & $\begin{array}{c}\text { Escore } \\
\text { mediano }\end{array}$ & $\begin{array}{c}\text { Erro } \\
\text { padrão }\end{array}$ & Valor de $p$ * \\
\hline \multicolumn{6}{|l|}{ Grau de afiliação ** } \\
\hline USF & 344 & 8,0 & 10 & 0,16 & \\
\hline UBS tradicional & 531 & 7,6 a & 10 & 0,12 & \\
\hline UBS mista & 609 & $8,3 b$ & 10 & 0,10 & $<0,001$ \\
\hline \multicolumn{6}{|l|}{ Acesso de primeiro contato - utilização } \\
\hline USF & 343 & 8,3 & 10 & 0,12 & \\
\hline UBS tradicional & 530 & 8,5 & 10 & 0,08 & \\
\hline UBS mista & 608 & 8,5 & 8,9 & 0,08 & 0,969 \\
\hline \multicolumn{6}{|c|}{ Acesso de primeiro contato - acessibilidade ** } \\
\hline USF & 342 & 5,0 a & 5,0 & 0,13 & \\
\hline UBS tradicional & 526 & $5,6 \mathrm{~b}$ & 6,7 & 0,12 & \\
\hline UBS mista & 580 & 5,2 a & 6,7 & 0,12 & 0,014 \\
\hline \multicolumn{6}{|l|}{ Longitudinalidade ** } \\
\hline USF & 343 & $6,5 b$ & 6,7 & 0,08 & \\
\hline UBS tradicional & 531 & 5,8 a & 6,0 & 0,07 & \\
\hline UBS mista & 609 & $6,3 \mathrm{~b}$ & 6,7 & 0,06 & $<0,001$ \\
\hline \multicolumn{6}{|l|}{ Coordenação - integração de cuidado ** } \\
\hline USF & 96 & 6,5 a & 8,0 & 0,35 & \\
\hline UBS tradicional & 154 & 7,0 & 8,0 & 0,27 & \\
\hline UBS mista & 177 & $7,5 \mathrm{~b}$ & 10 & 0,23 & 0,034 \\
\hline \multicolumn{6}{|c|}{ Coordenação - sistemas de informação ** } \\
\hline USF & 344 & 7,5 a & 7,8 & 0,11 & \\
\hline UBS tradicional & 531 & $7,8 \mathrm{~b}$ & 7,8 & 0,07 & \\
\hline UBS mista & 602 & 7,6 & 7,0 & 0,09 & $<0,001$ \\
\hline \multicolumn{6}{|l|}{ Integralidade - serviços disponíveis ** } \\
\hline USF & 282 & 5,2 a & 5,6 & 0,10 & \\
\hline UBS tradicional & 436 & $6,0 \mathrm{~b}$ & 6,7 & 0,11 & \\
\hline UBS mista & 499 & $6,7 c$ & 7,0 & 0,09 & $<0,001$ \\
\hline \multicolumn{6}{|l|}{ Integralidade - serviços prestados ** } \\
\hline USF & 343 & 5,3 a & 6,0 & 0,19 & \\
\hline UBS tradicional & 526 & $6,1 \mathrm{~b}$ & 6,7 & 0,15 & \\
\hline UBS mista & 600 & $6,9 c$ & 7,0 & 0,13 & $<0,001$ \\
\hline \multicolumn{6}{|l|}{ Orientação familiar ** } \\
\hline USF & 341 & $5,3 b$ & 5,6 & 0,16 & \\
\hline UBS tradicional & 526 & 4,4 a & 4,4 & 0,14 & \\
\hline UBS mista & 603 & $5,0 \mathrm{~b}$ & 3,3 & 0,13 & $<0,001$ \\
\hline \multicolumn{6}{|l|}{ Orientação comunitária ** } \\
\hline USF & 241 & $5,8 \mathrm{~b}$ & 5,8 & 0,19 & \\
\hline UBS tradicional & 398 & 4,9 a & 5,0 & 0,15 & \\
\hline UBS mista & 496 & $5,9 \mathrm{~b}$ & 6,7 & 0,13 & $<0,001$ \\
\hline
\end{tabular}

UBS: unidade básica de saúde; USF: unidade de saúde da família.

* Teste de Kruskal-Wallis (ANOVA one-way não paramétrica), resultado significativo valor de $p<0,05$;

** Teste de comparações múltiplas (post hoc) de Dunnett (subconjuntos homogêneos - escores médios não diferem estatisticamente entre si: a = a; subconjuntos heterogêneos - escores médios diferentes estatisticamente entre si: $a \neq b \neq c$ ). 
sistema de informação apresentaram forte presença e extensão nos três modelos de APS, ou seja, valores satisfatórios (escore $\geq 6,6$ ). $\mathrm{O}$ atributo coordenação-integração de cuidados foi superior ao ponto de corte nos modelos de UBS e UBS mista e integralidade - serviços disponíveis e serviços prestados foram alcançados somente pelo modelo de UBS mista.

Quanto aos atributos acesso de primeiro contato - acessibilidade, longitudinalidade, orientação familiar e orientação comunitária, os três modelos obtiveram escore inferior a 6,6. Entretanto, destaca-se que no modelo de USF, coordenação-integração de cuidados e longitudinalidade se aproximaram do ideal, com escore 6,5 (Tabela 4).

$\mathrm{Na}$ comparação entre os escores médios dos atributos, houve diferença estatisticamente significante entre USF, UBS e UBS mista para quase todos os atributos, salvo para acesso de primeiro contato - utilização. Contudo, entre os atributos que apresentaram diferença estatisticamente significativa, o modelo UBS mista tendeu a maiores escores para os atributos: grau de afiliação $(8,3)$, coordenação - integração de cuidados $(7,5)$, integralidade - serviços disponíveis $(6,7)$ e serviços prestados $(6,9)$ (Tabela 4).

\section{Discussão}

A análise dos resultados permitiu levantar a hipótese, apesar das limitações no controle de vieses do ponto de vista cultural, social, demográfico e de políticas públicas, que o modelo de UBS mista, quando comparado aos demais, apresentou diferença estatisticamente significativa e escore satisfatório à APS para a maioria dos atributos e também para o escore essencial, e foi o único com Escore Geral orientado à APS.

Esse dado merece atenção de todos os envolvidos no processo de cuidado à criança na APS, haja vista a conformação do modelo de UBS mista contar com a integração entre a UBS tradicional e a proposta de USF que, apesar de não ter alcançado isoladamente Escore Geral adequado à APS, chegou muito próximo disso com valor 6,4. Isso pode indicar que as fragilidades de cada modelo estão sendo supridas quando operam em conjunto, podendo estar influenciando o processo de cuidado à criança no modelo de UBS mista.

Estudos 16,17,18 que compararam distintos modelos de atenção em municípios brasileiros evidenciaram que tanto a USF quanto a UBS tradicional, dentre outros serviços de APS, não alcançaram Escore Geral adequado à atenção primária. Já outro 10 realizado no Paraná, evidenciou Escore Geral satisfatório de 6,6 para as unidades com ESF, enquanto a UBS tradicional esteve aquém do adequado, com escore 3,9 .

Diante disso, faz-se necessário refletir acerca das especificidades de cada modelo em relação às dimensões da APS, que podem estar contribuindo para qualificar a atenção à saúde da criança no modelo de UBS mista, bem como da implementação dos modelos nas diferentes regiões brasileiras, cujas especificidades podem estar comprometendo a atenção à criança.

Considerando individualmente os atributos da APS, pode-se sugerir que, independentemente do modelo, as famílias têm profissionais e serviços como fonte regular de atenção nas consultas de rotina para seguimento de necessidades de saúde já existentes ou novas demandas 6, incluindo encaminhamento para especialistas quando necessário, pois apresenta a dimensão acesso de primeiro contato - utilização satisfatória à APS e sem diferença entre os modelos cotejados, o que, isoladamente, é avaliado como positivo para a qualidade da atenção à criança na atenção primária.

Estudo realizado na China 19 evidenciou que os centros primários de saúde podem ser capazes de proporcionar um melhor atendimento de primeiro contato - utilização em relação a outros modelos existentes no país. No cenário nacional, estudo 10 comparativo evidenciou maior satisfação à APS nas unidades com ESF, cujo escore para utilização foi superior a 6,6 $(7,3)$ em relação às demais UBS tradicionais $(5,8)$. Isso pode indicar a influência da proposta de ESF no processo de trabalho dos profissionais que atuam na UBS mista como porta de entrada na rede de atenção à saúde, visto que as ações e serviços disponíveis no modelo de USF podem estar sendo mais bem organizados para atender às diferentes demandas da criança.

Por outro lado, quanto à dimensão acessibilidade, os dados evidenciaram baixos escores para todos os modelos, com diferença superior para UBS tradicional. Isso é preocupante do ponto de vista 
da inter-relação entre os atributos e qualidade do cuidado ofertado na APS, pois sugere que mesmo sendo considerada referência para a atenção à criança, as unidades que atuam com a proposta de ESF, como política de reorientação à APS 20, têm apresentado barreiras que dificultam o acesso aos serviços de APS, para a criança e sua família.

Além disso, é necessário identificar as possíveis potencialidades da UBS diante do processo de construção do cuidado à criança na UBS mista como modelo que compartilha o processo de trabalho dos profissionais da UBS tradicional em sua conformação de APS. Assim, ao se portar aos atendimentos realizados conforme demanda espontânea, constata-se que a UBS tradicional foi superior aos modelos que operam com a ESF em relação à acessibilidade. Isso sugere que nesse modelo as demandas da criança são acolhidas sem perder oportunidades, como preconiza a Política Nacional de Atenção Integral à Saúde da Criança 21.

Diante disso, faz-se premente reconhecer a importância da demanda espontânea frente ao risco de adoecimento da população, visto contribuir para formação de vínculo entre profissionais-serviçoscomunidade 4,22. Dessa maneira, articular demanda espontânea e programada pode contribuir para organização da APS como porta de entrada no sistema de saúde, como evidenciou estudo 23 no qual as equipes da UBS utilizam o acolhimento como estratégia para potencializar a coordenação do cuidado, enquanto a USF ainda tem incorporado ao processo de trabalho dos profissionais mecanismos tradicionais, como horários pré-estabelecidos para demanda espontânea, com maior facilidade de acesso para consulta programada.

Nesse ínterim, estudo 24 evidenciou que 80,6\% das consultas realizadas com crianças menores de 5 anos em USF foram sem agendamento, o que pode causar limitações no acompanhamento infantil de rotina e nas ações de prevenção e promoção à saúde, que culminaram em uma necessidade aguda de saúde. Porém, isso pode não implicar resolutividade do caso conforme evidenciou estudo30 acerca do controle das infecções respiratórias e de diarreia ofertado pela USF, porque foi considerado insatisfatório; e as explicações mais plausíveis permeiam, dentre outras, uma atuação médica centrada nas atividades clínicas, com deficit de atividades de educação em saúde para as famílias.

Quanto ao atributo integralidade, apenas a UBS mista apresentou alto escore à APS quanto aos serviços disponíveis (escore 6,7) e serviços prestados (escore 6,9), fato que pode indicar que na proposta da ESF, a integralidade, que se refere à prestação de um conjunto de serviços preventivos e curativos pelas unidades de saúde, ou encaminhados por elas de modo a atender às necessidades da população adscrita com capacidade de resposta aos problemas de saúde do indivíduo, em todos os ciclos de vida, possivelmente se encontra inadequada para a oferta de serviços à assistência à criança.

Por outro lado, tendo em vista as evidências apresentadas por estudo 18 comparativo em que, apesar das limitações dos modelos de UBS tradicional e ESF na assistência à criança frente às demandas mais comuns de saúde, a UBS se destacou com maior disponibilidade e melhores serviços prestados. Fatores como melhores estruturas, presença diária de pediatra e melhor oferta de atividades, como imunização e promoção à saúde em grupo, podem ser aspectos diferenciais para o alcance da integralidade da atenção à criança pela UBS mista.

No entanto, ao levar em conta o grau de afiliação, o fato de a UBS mista ter apresentado diferença estatisticamente significativa superior apenas à UBS tradicional, sugere que os princípios do modelo de saúde da família podem ter contribuído para esse resultado, conforme evidenciou estudo 10 sobre o melhor desempenho das unidades que operam com a ESF (escore 9,0) em relação à UBS tradicional $(1,7)$ para tal dimensão. Isso pode ocorrer pelo potencial da ESF para considerar o indivíduo um ser social no processo saúde-doença, contrariando o que se preconiza em relação à fonte habitual de atenção à saúde, que precisa estar orientada para a pessoa e não para a doença 7 , de modo que os usuários e prestadores de serviços se reconheçam na inter-relação que se estabelece no processo de produção de saúde e continuidade do cuidado, para que assim, seja garantida a longitudinalidade da atenção na APS.

Com relação à longitudinalidade, nenhum modelo foi satisfatório à APS, concordando com outros estudos 10,18,25. Porém, os modelos que operam com a ESF, isto é, a USF $(6,5)$ e UBS mista $(6,3)$, apresentaram escore mais próximo do ponto de corte em relação à UBS tradicional $(5,8)$. Assim também se apresentaram os atributos derivados, sendo a orientação familiar com diferença superior para USF e a orientação comunitária para UBS mista. Pode-se sugerir uma maior regularidade desses modelos no seguimento da atenção à criança na APS, bem como reconhecimento pelos profissionais do contexto 
familiar, comunitário e social da criança assistida. Estudo 22 que analisou fragilidades e limitações da ESF e da UBS quanto às dimensões da APS, evidenciou que o vínculo, a longitudinalidade e a orientação familiar e orientação comunitária 10 ocorreram de forma mais efetiva na USF, segundo a visão dos usuários.

Além do mais, estudo 26 destacou que dentre os fatores associados ao melhor desempenho da APS está a formação dos profissionais em saúde da família, a disponibilidade do médico na equipe 30 horas semanais, bem como o número de equipes por UBS, o que faz emergir a necessidade de ampliação das UBS, a fim de acomodar mais equipes de ESF e possibilitar maior integração, diálogo e troca de experiências entre elas, rumo à qualidade do cuidado na APS.

Essa realidade é positiva, uma vez que o modelo USF, como proposta de reorientação da APS, mesmo com limitações em reconhecer a criança como sujeito integral no processo saúde-doença ao longo do tempo pelos escores inferior a 6,6 10,18, pode estar se destacando em relação ao modelo tradicional, para a continuidade do atendimento à criança pelo mesmo profissional (escore 7,0), ao tempo em que está entendendo os questionamentos da família (escore 7,3) 27. Isso indica que o profissional que atua nesse modelo pode estar concentrando esforços para adequar sua prática em consonância com a APS.

Ressalta-se que a proposta da ESF na atenção à criança busca promover o acompanhamento sistemático do crescimento e desenvolvimento infantil, fortalecendo o vínculo e a corresponsabilidade entre profissionais e família para o alcance de resolutividade do cuidado diante dos problemas dessa população 28 . Mas, para tanto, a fonte cuidadora precisa cumprir com o papel de coordenadora do cuidado e a garantia de uma atenção integral à criança na APS 18, o que ainda se configura um desafio.

A coordenação do cuidado que é entendida como a capacidade de organizar os serviços em prol da garantia de continuidade do cuidado à saúde na rede de atenção ${ }^{29}$, precisa ir além do encaminhamento, promovendo a comunicação entre os profissionais e serviços com garantia de referência e contrarreferência (integração de cuidados), compartilhamento das informações geradas pelos profissionais na oferta de serviços à criança (sistema de informação) desde a porta de entrada e de volta para ela, de modo que ocorram a continuidade do cuidado e a resolutividade na APS 6.

No estudo em tela, dois achados chamaram a atenção, o fato de a UBS mista ter apresentado diferença significativa em relação à USF para o atributo coordenação - integração de cuidados, e a UBS tradicional se destacar em relação aos outros dois modelos para dimensão sistema de informação. Isso pode demonstrar que, apesar das experiências exitosas das unidades com ESF diante da maioria das referências a serviços especializados e, portanto, melhor organização dos fluxos para a atenção especializada 10,17, possíveis entraves dificultam seu papel de ordenadora da rede e coordenadora da atenção, como falta de regulação e de fluxos formais para atenção hospitalar 29 e oferta limitada de serviços de maior densidade tecnológica para diagnóstico, tratamento e resolutividade. Esses aspectos podem estar comprometendo o acesso e a integração dos serviços, repercutindo no retorno dos usuários à porta de entrada e gerando demanda reprimida com baixa resolutividade 22.

Faz-se necessário repensar o fato de o modelo UBS tradicional dispor na equipe de profissionais especialistas, como pediatras, que podem estar atendendo com mais resolutividade às demandas infantis na APS. Aponta-se que tanto as mães depositam maior credibilidade e confiança nos especialistas, em relação aos médicos generalistas da USF para atenderem às suas crianças, como também os próprios pediatras alegam a insegurança de colegas de outras áreas em atender às especificidades da criança na USF 3 .

Os resultados ora apresentados acerca da oferta e qualidade da atenção à criança, consonantes com os atributos da APS, podem ser interpretados com limitações, já que a entrevista foi realizada no âmbito das unidades de saúde nas quais os profissionais atuam, situação que pode ter constrangido o familiar e ou cuidador quanto à liberdade de opção nas respostas, bem como o fato de ter considerado apenas a visão do usuário, que comumente difere da dos profissionais.

Contudo, observou-se que os achados, apesar da limitação no ajuste dos vieses apontados, levantam a hipótese de que a UBS mista, que mescla ESF e cuidado especializado, nesse caso, do pediatra, é o mais orientado à APS na atenção à criança, dentre os três modelos estudados. Talvez isso se relacione ao fato de que os diferentes modelos avaliados pertenciam a municípios diferentes, sem possibilidade de ajustar, na análise, as variáveis ligadas aos diferentes contextos. Portanto, estudos futuros, com desenhos mais robustos, e ampliando para outros usuários e profissionais de saúde, possam comprovar esta hipótese. 


\section{Colaboradores}

N. C. C. B. Santos, B. R. G. O. Toso e A. P. S. Reichert participaram da concepção e projeto, análise e interpretação dos dados, e contribuíram com a redação do artigo, revisão crítica relevante do conteúdo intelectual e aprovação final da versão a ser publicada. E. M. C. Vaz, J. A. Nogueira e N. Collet contribuíram com redação do artigo, revisão crítica relevante do conteúdo intelectual e aprovação final da versão a ser publicada.

\section{Agradecimentos}

Os autores agradecem aos familiares e/ou cuidadores que participaram da pesquisa e contribuíram com a investigação; ao Conselho Nacional de Desenvolvimento Científico e Tecnológico - CNPq (Edital universal 014/2011) e a bolsa de produtividade à Neusa Collet.

\section{Referências}

1. Mello DF, Furtado MCC, Fonseca LMM, Pina JC. Child health follow-up and the longitudinality of caring. Rev Bras Enferm 2012; 65:675-9.

2. Novaczyk AG, Gaíva MAM. Interrelational technologies on the child healthcare: analysis of official documents. Ciênc Cuid Saúde 2010; 9:560-8.

3. Sousa FGM, Erdman AL, Mochel EG. Limiting conditions for intregal care for a child in Brazilian Primary Health Care. Texto \& Contexto Enferm 2011; 20(Esp):263-71.

4. Finkler AL,Viera CS, Tacla MTGM, Toso BRGO. The access and the difficulty in resoluteness of the child care in primary health care. Acta Paul Enferm 2014; 27:548-53.

5. Oliveira MAC, Pereira IC. Primary health care essential attributes and the Family Health Strategy. Rev Bras Enferm 2013; 66(Esp): 158-64.
6. Starfield B. Atenção primária: equilíbrio entre necessidades de saúde, serviços e tecnologia. Brasília: Organização das Nações Unidas para a Educação, Ciência e a Cultura/Ministério da Saúde; 2002.

7. Starfield B, Shi L, Macinko J. Contribution of primary care to heath systems and health. Milbank Q 2005; 83:457-502.

8. Harzheim E, Oliveira MMC, Agostinho MR, Hauser L, Stein AT, Gonçalves MR, et al. Validação do instrumento de avaliação da atenção primária à saúde: PCATool-Brasil adultos. Rev Bras Med Fam Comunidade 2013; 8:274-84.

9. Mendes EV. A construção Social da atenção primária à saúde. Brasília: Conselho Nacional de Secretários de Saúde; 2014.

10. Oliveira VBCA, Veríssimo MLOR. Children's health care assistance according to their families: a comparison between models of Primary Care. Rev Esc Enferm USP 2015; 49:30-6. 
11. Plano Municipal de Saúde 2014-2017. http://www.londrina.pr.gov.br/index. php?option $=$ com_content $\&$ view $=$ article $\&$ id $=$ $567 \&$ Itemid=613 (acessado em 20/Nov/2015).

12. Agencia Nacional de Vigilância Sanitária. Resolução RDC no 50, de 21 de fevereiro de 2002. Dispõe sobre regulamento técnico para planejamento, programação, elaboração e avaliação de projetos físicos de estabelecimentos assistenciais de saúde. Diário Oficial da União 2002; 20 mar.

13. Starfield B, Cassady CE, Hurtado MP, Berk RA, Nanda JP, Friedenberg LA. Measuring consumer experiences with primary care. Pediatrics 2000; 105:998-1003.

14. Harzheim E, Starfield B, Rajmil L, ÁlvarezDardet C, Stein AT. Consistência interna e confiabilidade da versão em português do Instrumento de Avaliação da Atenção Primária (PCATool-Brasil) para serviços de saúde infantil. Cad Saúde Pública 2006; 22:1649-59.

15. Departamento de Atenção Básica, Secretaria de Atenção em Saúde, Ministério da Saúde. Manual do instrumento de avaliação da atenção primária à saúde: primary care assessment tool pcatool - Brasil. Brasília: Ministério da Saúde; 2010. (Série A. Normas e Manuais Técnicos).

16. Leão CDA, Caldeira AP, Oliveira MMC. Atributos da atenção primária na assistência à saúde da criança: avaliação dos cuidadores. Rev Bras Saúde Matern Infant 2011; 11:323-34.

17. Mesquisa Filho M, Luz BSR, Araújo CS. Primary health care and its atributes: the situation of children under two years of age according to their caregivers. Ciênc Saúde Coletiva 2014; 19:2033-46.

18. Araújo RL, Mendonça AVM, Sousa MF. Percepção dos usuários e profissionais de saúde do Distrito Federal: os atributos da atenção primária. Saúde Debate 2015; 39:387-99.

19. Wangi HHW, Wong SYS, Wong MCS, Wang JJ, Wei XL, Li DKT, et al. Attributes of primary care in community health centres in China and implications for equitable care: a cross-sectional measurement of patients' experiences. QJM 2015; 108:549-60.

20. Ribeiro LCC, Rocha RL, Ramos-Jorge ML. Acolhimento às crianças na atenção primária à saúde: um estudo sobre a postura dos profissionais das equipes de saúde da família. Cad Saúde Pública 2010; 26:2316-22.
21. Ministério da Saúde. Portaria GM no 1.130, de 05 de agosto de 2015. Política Nacional de Atenção integral à Saúde da Criança. Diário Oficial da República Federativa do Brasil 2015; 5 ago.

22. Marin MJS, Marchioli M, Maracvick MYA. Fortalezas e limitações do atendimento nas unidades básicas de saúde tradicionais e da estratégia de saúde da família pela ótica dos usuários. Texto \& Contexto Enferm 2013; 22:780-8

23. Almeida PF, Faust MCR. Fortalecimento da atenção primária à saúde: estratégia para potencializar a coordenação dos cuidados. Rev Panam Salud Pública 2011; 29:84-95.

24. Ribeiro JM, Siqueira SAV, Pinto LFS. Children health care evaluation ( $0-5$ years) according to users' perceptions in the Family Health Strategy of Teresópolis, Rio de Janeiro State. Ciênc Saúde Coletiva 2010; 15:517-27.

25. Vitória AM, Harzheim E, Takeda SP, Hauser L. Avaliação dos atributos da atenção primária à saúde em Chapecó, Brasil. Rev Bras Med Fam Comunidade 2013; 8:285-93.

26. Turci MA, Lima-Costa MF, Macinko J. The influence of structural and organizational factors on the performance of primary health care in Belo Horizonte, Minas Gerais State, Brazil, according to nurses and managers. Cad Saúde Pública 2015; 31:1941-52.

27. Oliveira VC, Veríssimo MLOR. A prática da longitudinalidade no atendimento à saúde da criança: comparação entre modelos assistenciais distintos. Cogitare Enferm 2015; 20:45-52.

28. Souza RS, Ferrari RAP, Santos TFM, Tacla MTGM. Pediatric health care: practice of nurses in the Family Health Program. REME Rev Min Enferm 2013; 17:340-8.

29. Almeida PF, Giovanella L, Mendonça MHM, Escorel S. Desafios da coordenação dos cuidados em saúde: estratégias de integração entre níveis assistenciais em grandes centros urbanos. Cad Saúde Pública 2010; 26:286-98. 


\section{Abstract}

The aim of this study is to compare traditional primary care units and family health units to determine which of the two models is better oriented towards primary care for children, considering the existence and scope of essential and intermediate attributes of primary care. We interviewed a total of 1,484 parents and guardians of children under 10 years of age enrolled in family health units in João Pessoa, Paraíba State, Brazil, as well as in traditional primary care units in Cascavel, Paraná State, Brazil, and mixed primary care units in Londrina, Paraná State, Brazil. The Primary Care Assessment Tool Brazil, children's version, was used for this purpose. The parametric one-way ANOVA test was used to identify statistically significant differences between the models of care, followed by the Tukey post hoc multiple comparison test to identify which model presented differences. The study design's limitations notwithstanding, the findings raised the hypothesis that mixed primary care units were the model best oriented to primary care for children. The difference may relate to the fact that the different models belonged to different municipalities, without the possibility of adjusting the variables linked to the different contexts in the variables. Thus, this hypothesis may be proven by future studies with more robust designs and expanded to include other users and health professionals.

Primary Health Care; Child Health; Health Services Research

\section{Resumen}

El objetivo de este estudio es evaluar cuál es el modelo de atención, entre la unidad básica de salud tradicional y aquellas con Estrategia de Salud de la Familia, que está más orientado a la atención primaria a la salud (APS) del niño, considerando la presencia y la extensión de las particularidades esenciales y derivadas de la APS. Fueron entrevistados 1.484 familiares $y / o$ cuidadores de niños menores de 10 años de edad, registrados en unidades de salud de la familia de João Pessoa en Paraíba, Brasil, y unidades básicas de salud tradicionales de Cascavel, y unidades básicas de salud mixtas de Londrina, ambas en Paraná, Brasil. Para ello, se utilizó el Primary Care Assessment Tool Brasil, versión niños. Para el análisis se utilizó el test ANOVA one way paramétrico, con el fin de identificar las diferencias estadisticamente significativas entre los modelos de atención, seguidos del test de comparación múltiple (post hoc) de Tukey, para apuntar qué modelo presentaba diferencias. A pesar de las limitaciones en el diseño del estudio, los hallazgos sugieren la hipótesis de que la unidad básica de salud mixta es el modelo más orientado a la APS en el cuidado al niño. Esto puede estar relacionado con el hecho de que los diferentes modelos evaluados pertenecían a municipios diferentes, sin posibilidad de ajustar, en el análisis, las variables relacionadas con los diferentes contextos. Así, estudios futuros, con diseños más robustos, si se amplian a otros usuarios y profesionales de salud, podrían comprobar esta hipótesis.

Atención Primaria de Salud; Salud del Niño; Investigación en Servicios de Salud
Recebido em 27/Jan/2016

Versão final reapresentada em 03/Mai/2017 Aprovado em 12/Mai/2017 